\title{
Variación anatómica en la madera de Quercus obtusata (Fagaceae)
}

\author{
Dulce María Chávez-Romero1, Silvia Aguilar-Rodríguez¹ \\ y Teresa Terrazas 2
}

\section{Anatomical variation in Quercus obtusata (Fagaceae) wood}

\begin{abstract}
RESUMEN
Se describen las características anatómicas de la madera de Quercus obtusata y se evalúa estadísticamente la variación anatómica de nueve caracteres cuantitativos de su madera en función de la altura y diámetro del tallo, de la altitud y de 11 parámetros climáticos en cuatro localidades. La correlación de Pearson mostró que sólo la longitud del elemento de vaso angosto se asocia significativamente con la altura del árbol $\left(r_{p}=0,70, p<0,02\right)$. El análisis de componentes principales reveló que en tres componentes se explica $76,46 \%$ de la variación total y diez variables son las más importantes. Este análisis segregó las poblaciones debido a la altitud y algunas variables climáticas, siendo la variación de la temperatura y la precipitación a lo largo del año, las que tuvieron un efecto sobre la longitud de los elementos de vaso, así como sobre la pared y longitud de la fibra. En la localidad más seca, Arcos del Sitio, y en la de mayor altitud, Santa Ana Jilotzingo, los elementos de vaso con mayor diámetro son más cortos (561 um y $552 \mu \mathrm{m}$ respectivamente) que los del ambiente más húmedo, Santa Catarina (621 $\mu \mathrm{m})$. Las fibras no disminuyen su tamaño con la altitud ni lo aumentan con la talla de los individuos. Este análisis sugiere que se debe tomar en cuenta la procedencia microclimática de las poblaciones para hacer recomendaciones de uso de la madera de $Q$. obtusata o para planes de reforestación.
\end{abstract}

PALABRAS CLAVE:

Componentes principales, correlación anatómica, elementos de vaso, gradiente ambiental.

\begin{abstract}
Wood of Quercus obtusata is described and the variation of nine quantitative wood characters is evaluated through tree height and diameter, elevation and 11 climatic parameters in four sites. Pearson correlation showed that only the narrow vessel element length is significantly correlated with tree height $\left(r_{p}=0,70, p<0,02\right)$. The principal component analysis revealed that three components explain $76,46 \%$ of the total variation and ten variables are those with the highest loadings. Moreover, this analysis segregated the localities due to altitude and some climatic variables as for temperature and rainfall throughout the year, which have an effect on vessel elements length, as well as on fibres length and wall thickness. The driest locality, Arcos del Sitio, and in that of higher elevation, Santa Ana Jilotzingo, have the shortest vessel elements with wider diameter (561 $\mu \mathrm{m}$ and $552 \mu \mathrm{m}$ respectively) than the most humid environment, Santa Catarina $(621 \mu \mathrm{m})$. The fibres neither diminish in size with the elevation nor increase with tree height. The analyses suggest that the microclimatic parameters of each location need to be accounted when recommendations for Q. obtusata wood used as raw material or in reforestation programs are given.
\end{abstract}

KEY WORDS:

Principal components, anatomical correlation, vessel elements, environmental gradient. y Función. Av. de los Barrios no. 1. Estado de México 54090. correo-e siagro@campus.iztacala.unam.mx Universidad Nacional Autónoma de México (UNAM). Instituto de Biología. Apartado Postal 70-233, 04510 México, D.F. correo-e tterrazas@ibiologia.unam.mx. 


\section{INTRODUCCIÓN}

Quercus obtusata Humboldt \& Bonpland incluye árboles que miden entre 6 y 30 metros de alto, con diámetros a la altura del pecho hasta de $60 \mathrm{~cm}$. Es una de las especies de encino con más amplia distribución en nuestro país (Valencia, 2004), registrándose de Nuevo León, Durango y Tamaulipas hasta Guerrero y Oaxaca. Prospera en bosques de Pinus, Quercus, Pinus-Quercus y bosque mesófilo de montaña, asociado con otras especies. También se le encuentra en el pastizal con matorral xerófilo de Acacia y Opuntia, así como en encinares perturbados; en altitudes que fluctúan entre los 1430 y 2850 m (De la Paz Pérez-Olvera et al., 2006; Romero et al., 2002; Rzedowski, 1978). Por su alta densidad básica es una especie con un alto potencial de aprovechamiento (Honorato y Fuentes, 2001). Su madera es excelente para ser usada como duela para pisos y es adecuada para tonelería; se emplea en la elaboración de leña, carbón, postes para cercas, implementos agrícolas, cabos para herramienta y como material de construcción (De la Paz Pérez-Olvera, 1982; De la Paz Pérez-Olvera et al., 2000; Ortega et al., 1988; Quintanar, 2002; Romero et al., 2002). A pesar de la gran importancia que la madera de $Q$. obtusata tiene como recurso forestal, no existen estudios sobre su variación anatómica en nuestro país. En regiones de Europa y América del Norte, toman en cuenta el origen geográfico de las especies con la finalidad de establecer un estricto criterio de selección sobre sus poblaciones, pues se ha registrado que existen diferencias significativas en la dimensión de los elementos celulares en la madera de algunos encinos asociadas con el clima en el que sus individuos crecen (VillaSalvador et al., 1997). En México este tipo de trabajos son escasos (AguilarRodríguez et al., 2006; Arias y Terrazas, 2001; Terrazas y Loza-Cornejo, 2003) y para los encinos no se reportan. La literatura hace referencia a estudios anatómicos de la madera realizados en varias especies de Quercus (Aguilar- Rodríguez et al., 2000; Aguilar- Rodríguez y CastroPlata, 2006; De la Paz Pérez-Olvera, 1976, 1982; De la Paz Pérez-Olvera y Dávalos-Sotelo, 2008; De la Paz PérezOlvera y Quintanar, 1994; De la Paz Pérez-Olvera et al., 1998, 2006; Corral, 1981; Martínez et al., 2003; Valencia y Barajas-Morales, 1995) y algunos de éstos incluyen a $Q$. obtusata (AguilarRodríguez et al., 2000; De la Paz PérezOlvera, 1982, 1985; De la Paz PérezOlvera y Dávalos-Sotelo, 2008; De la Paz Pérez-Olvera et al., 2006; Ortega et al., 1988), pero en ninguno se correlacionan los caracteres de la madera con factores climáticos, la altitud o con la altura y diámetro de los árboles.

Los estudios sobre variación anatómica de madera en especies de importancia económica proveen información que puede ser usada como registro de las condiciones ambientales donde los individuos se desarrollan y son fundamentales ya que proveen las bases para un manejo forestal adecuado que favorezca las plantaciones o la restauración regional.

\section{OBJETIVOS}

En este trabajo se describen las características anatómicas de la madera de Quercus obtusata y se evalúa a través de un análisis estadístico la variación de nueve caracteres cuantitativos en función de 11 parámetros climáticos y la altitud, así como con la altura y diámetro de los árboles que crecen bajo diferentes condiciones climáticas.

\section{METODOLOGÍA}

Colecta. Se recolectaron muestras de $Q$. obtusata provenientes de cuatro locali- 
dades en diferentes ambientes (Tabla 1). En estos sitios el clima varía de templado a seco, con una temperatura que fluctúa entre los $12^{\circ}$ y $20^{\circ} \mathrm{C}$ y precipitación promedio anual entre $711 \mathrm{~mm}$ y $1463 \mathrm{~mm}$ (García, 2004). La vegetación predominante de acuerdo a Rzedowski (1978) en las localidades de colecta, correspondió a bosque de encino-pino, encino, encino con elementos mesófilos y matorral xerófilo con diferentes grados de perturbación. Se recolectaron ejemplares de herbario para respaldo, éstos fueron depositados en el herbario IZTA. En el campo se obtuvieron datos generales como la altura y el diámetro de los árboles a la altura del pecho. Los sitios fueron georeferenciados con un GPS Magellan modelo Mapa 410. En cada localidad se muestrearon tres individuos maduros y sanos, excepto en Santa Ana Jilotzingo en donde fueron dos. A una altura de $1,30 \mathrm{~m}$ del tronco se obtuvo una troza $(2-3 \mathrm{~cm}$ de alto $\times 7 \mathrm{~cm}$ de ancho x 5-7 cm de largo) empleando un serrote, cincel y martillo. Las trozas se fijaron en una solución de formol - ácido acético - alcohol al 70\% (Johansen, 1940) por $48 \mathrm{~h}$ y posteriormente se ablandaron en una solución de glicerina-alcohol etílico-agua (1:2:3) por tres meses.

Trabajo de laboratorio. De cada una de las trozas se obtuvieron cubos de $1,5 \mathrm{~cm}$ de lado y con un micrótomo de deslizamiento se cortaron en los planos transversal, tangencial y radial, obteniendo secciones de 20 a $30 \mu \mathrm{m}$ de grosor. Las secciones se tiñeron con safranina y posteriormente se montaron de acuerdo a la técnica propuesta por Johansen (1940). Con ayuda de un microscopio estereoscópico y usando una navaja desechable del micrótomo, se separó la madera temprana de la tardía de los primeros cuatro anillos de crecimiento cercanos a la corteza. Las astillas de la madera temprana se colocaron en la solución Jeffrey (Berlyn y Miksche, 1976) con la finalidad de obtener material disociado y medir la longitud de los elementos de vaso ancho y angosto y la de las fibras, así como identificar la presencia de traqueidas vasicéntricas.

Descripción y análisis estadístico. Se llevó a cabo la descripción anatómica de la madera según lo recomendado por la Asociación Internacional de los Anatomistas de la Madera (IAWA Committee, 1989). Se tomaron 50 mediciones para diámetro del lumen de vaso ancho (> 150 $\mu \mathrm{m})$ y angosto $(\leq 150 \mu \mathrm{m})$, longitud del elemento de vaso ancho (> $150 \mu \mathrm{m})$ y angosto $(\leq 150 \mu \mathrm{m})$; longitud, diámetro del lumen, diámetro total y grosor de la pared de las fibras, diámetro de las punteaduras intervasculares y altura del radio uniseriado. Los radios multiseriados no se cuantificaron debido al tamaño pequeño de los trozas. Con ellos se obtuvo la estadística descriptiva (media, desviación estándar y valores mínimo y máximo) (Tabla 2). Con los valores promedio de los caracteres grosor de pared y diámetro del lumen de las fibras, se calculó la Relación Runkel $(\mathrm{RR}=2 \mathrm{w} / \mathrm{l}$; donde $\mathrm{w}=$ grosor de pared de fibra y I=diámetro de lumen de fibra) para cada localidad. Los caracteres se describieron y cuantificaron empleando un analizador de imágenes NISElements BR 2.33 (Nikon Corporation, 1991-2006).

Con base en las localidades georeferenciadas para cada población, se generaron 18 parámetros climáticos mediante el uso del programa de modelaje bioclimático (Bioclim versión 2.0; Busby, 1986). Debido a que la madera tardía en algunos casos no fue evidente o en otros estaba escasamente desarrollada, exclusivamente se incluyeron en los análisis estadísticos nueve caracteres cuantititativos de la madera temprana. La correlación de Pearson se obtuvo entre los caracteres de la madera con la altura 
y diámetro del árbol y la altitud. Considerando la alta autocorrelación entre algunas de las 18 variables climáticas, únicamente 11 no autocorrelacionadas significativamente se analizaron en conjunto con nueve variables de la madera temprana, la altura y diámetro de los árboles y la altitud mediante un análisis de componentes principales (ACP). El ACP se realizó con la finalidad de identificar que parámetros contribuían significativamente a explicar el mayor porcentaje de la variación en la madera de $Q$. obtusata. A las nueve variables de la madera temprana y la Relación Runkel se les aplicó un análisis de varianza del modelo general lineal, seguido por un análisis de comparación de medias (Duncan, 0.05) para detectar diferencias significativas entre localidades. Los análisis estadísticos se llevaron a cabo empleando el programa estadístico Statistical Analysis System (SAS Institute, 1989).

\section{RESULTADOS}

\section{Descripción anatómica (Figuras 1-3)}

Anillos de crecimiento delimitados en la madera tardía por la presencia de bandas de fibras con forma rectangular en vista transversal y las paredes engrosadas, además de algunos vasos con un diámetro menor que el de los vasos de la madera temprana. Se reconocen tres variantes en las bandas de fibras que conforman la madera tardía: (1) Bandas apenas perceptibles $o$ angostas formadas por 3-5 hileras de células (Figs. 1a, 2c), (2) bandas angostas formadas por 3-5 hileras de células, asociadas con bandas anchas de fibras de forma redondeada $y$ pared gruesa (Fig. 1c) y (3) bandas en las que el grosor de la pared de las fibras disminuye gradualmente hacia la madera temprana (Fig. 2a). Porosidad difusa
(Figs. 1, 2); vasos principalmente solitarios, arreglados en cadenas diagonales. Diámetro tangencial promedio de los vasos anchos en la madera temprana 195 $\pm 21 \mu \mathrm{m}(169-232 \mu \mathrm{m})$ y de $63 \pm 25 \mu \mathrm{m}$ (15-141 $\mu \mathrm{m})$ en los vasos angostos de la madera tardía. Se observan en la madera temprana algunos vasos con diámetro angosto, similar a los de la madera tardía. Longitud promedio de los elementos de vaso anchos $\mathrm{y}$ angostos en la madera temprana $580 \pm 59 \mu \mathrm{m}(508-722 \mu \mathrm{m}) \mathrm{y}$ $605 \pm 43 \mu \mathrm{m}(514-666 \mu \mathrm{m})$ respectivamente; placas de perforación simple con una inclinación de 10 a $45^{\circ}$, punteaduras intervasculares alternas $9 \pm 0.54 \mu \mathrm{m}$ (8.4$10.2 \mu \mathrm{m})$, punteaduras vaso-radio de bordes reducidos a aparentemente simples; en algunos casos con tílides en vasos. Fibras libriformes con una longitud promedio de $1477 \pm 106 \mu \mathrm{m}$ (1303-1618 $\mu \mathrm{m})$, diámetro total de $21,8 \pm 4,3 \mu \mathrm{m}$ (17,8-34,5 $\mu \mathrm{m})$, diámetro del lumen de 6,7 $\pm 0,5 \mu \mathrm{m}(5,6-7,6 \mu \mathrm{m})$ y paredes gruesas con $6,2 \pm 0,8 \mu \mathrm{m}(5,0-7,6 \mu \mathrm{m})$; presencia de traqueidas vasicéntricas. Parénquima axial apotraqueal y paratraqueal, de abundante a regular; el apotraqueal difuso y en agregados, formando bandas tangenciales y en algunos casos diagonales; el paratraqueal vascicéntrico (Figs. 1, 2); en series de 4 a 8 células; cristales prismáticos ocasionales y granos de almidón (Fig. 3a-c). Radios uniseriados y multiseriados homogéneos (Fig. 3d, e), altura de los radios uniseriados de $464 \pm$ $108 \mu \mathrm{m}$ (241-614 $\mu \mathrm{m})$, con células procumbentes; en algunos casos con cristales romboidales en pocas células.

\section{Análisis estadísticos}

Correlación entre caracteres anatómicos. En la tabla 3 se sintetizan los coeficientes de correlación significativos entre los nueve caracteres anatómicos. Las correlaciones más altas y positivas se presentaron entre la longitud de los elementos 
de vaso ancho y angosto y entre la longitud del elemento de vaso ancho y la longitud de las fibras; una correlación más baja existió entre la longitud del elemento de vaso angosto y la altura del radio.

Correlación de los caracteres anatómicos con la altura y diámetro de los individuos $y$ altitud. No se encontraron correlaciones significativas entre los caracteres anatómicos de la madera y el tamaño de los árboles $\left(r_{p s}-0,19-0,42, p>0,54\right)$, excepto para la longitud del elemento de vaso angosto $\left(r_{p}=0,70, p<0,02\right)$. No existe correlación significativa entre la altitud con alguna de las variables de la madera $\left(r_{p s}<-0,56, p>0,06\right)$.

Con respecto a las variables relacionadas con las dimensiones de los árboles el análisis de correlación mostró que el tamaño se asocia positivamente con el diámetro del tronco $\left(r_{p}=0,82 p<0,002\right)$; a su vez este último tiene una correlación significativa y negativa con la altitud $\left(r_{p}=\right.$ $-0,79, p<0,003)$.

Análisis de Componentes Principales. El análisis de componentes principales mostró que los tres primeros componentes explican $76,46 \%$ de la variación total de la madera de $Q$. obtusata (Tabla 4). El primer componente explicó $35,88 \%$ de la varianza total y los parámetros climáticos que presentaron mayor peso son las variables de la temperatura [temperatura promedio del periodo más frío (TPF) y la temperatura del periodo más cálido (TPC)] y la altitud. El segundo componente explicó $26,61 \%$ de la varianza y son la distribución de la precipitación a lo largo del año [precipitación anual (PA), precipitación del cuatrimestre más cálido (PCC) y precipitación del cuatrimestre más frío (PCF)] los que más contribuyeron. El tercer componente explicó $13,96 \%$ de la varianza remanente y tres caracteres anatómicos tuvieron mayor peso: la longitud de la fibra, longitud del elemento de vaso ancho y el grosor de la pared de la fibra, así como la relación pared/lumen de la fibra (Relación Runkel).

La figura 4 muestra la representación gráfica de los individuos de cada población, donde el primer componente separa hacia la derecha a la población de Santa Catarina, cuyos individuos se desarrollan a una menor altitud, en un ambiente donde la temperatura en el transcurso del año es la más alta de las cuatro poblaciones, con un promedio de temperaturas en las épocas cálida fría de 19,2 y $14,9^{\circ} \mathrm{C}$ respectivamente. En estas condiciones, las maderas presentaron los elementos de vaso más largos, así como el mayor diámetro del lumen de la fibra y correspondieron a los individuos de mayor talla. Por el contrario, hacia el lado izquierdo se separaron las poblaciones que se desarrollan en sitios con temperaturas en general más bajas que las de Santa Catarina. En estas poblaciones la longitud promedio de los elementos de vaso fue menor y también corresponden a individuos de menor talla. El segundo componente separa hacia la parte superior de la gráfica a la población de Santa Ana Jilotzingo debido a que en la zona existe una mayor precipitación promedio anual, con $1085 \mathrm{~mm}$ y en los periodos más cálido y más frío llueve alrededor de 312 y 38 mm respectivamente; pero esta localidad se encuentra a una altitud de $2700 \mathrm{~m}$, que es la mayor registrada de los cuatro sitios, asimismo es la que muestra los valores más bajos de temperatura a lo largo del año. En estas maderas se reconocen los elementos de vaso ancho más cortos $(552,30 \pm 73 \mu \mathrm{m})$ y fibras con longitud y diámetro total más pequeños $(1428,43 \pm 185 \mu \mathrm{m}$ y $20,65 \pm$ $2 \mu \mathrm{m}$ respectivamente) de las cuatro localidades (Tabla 2). 


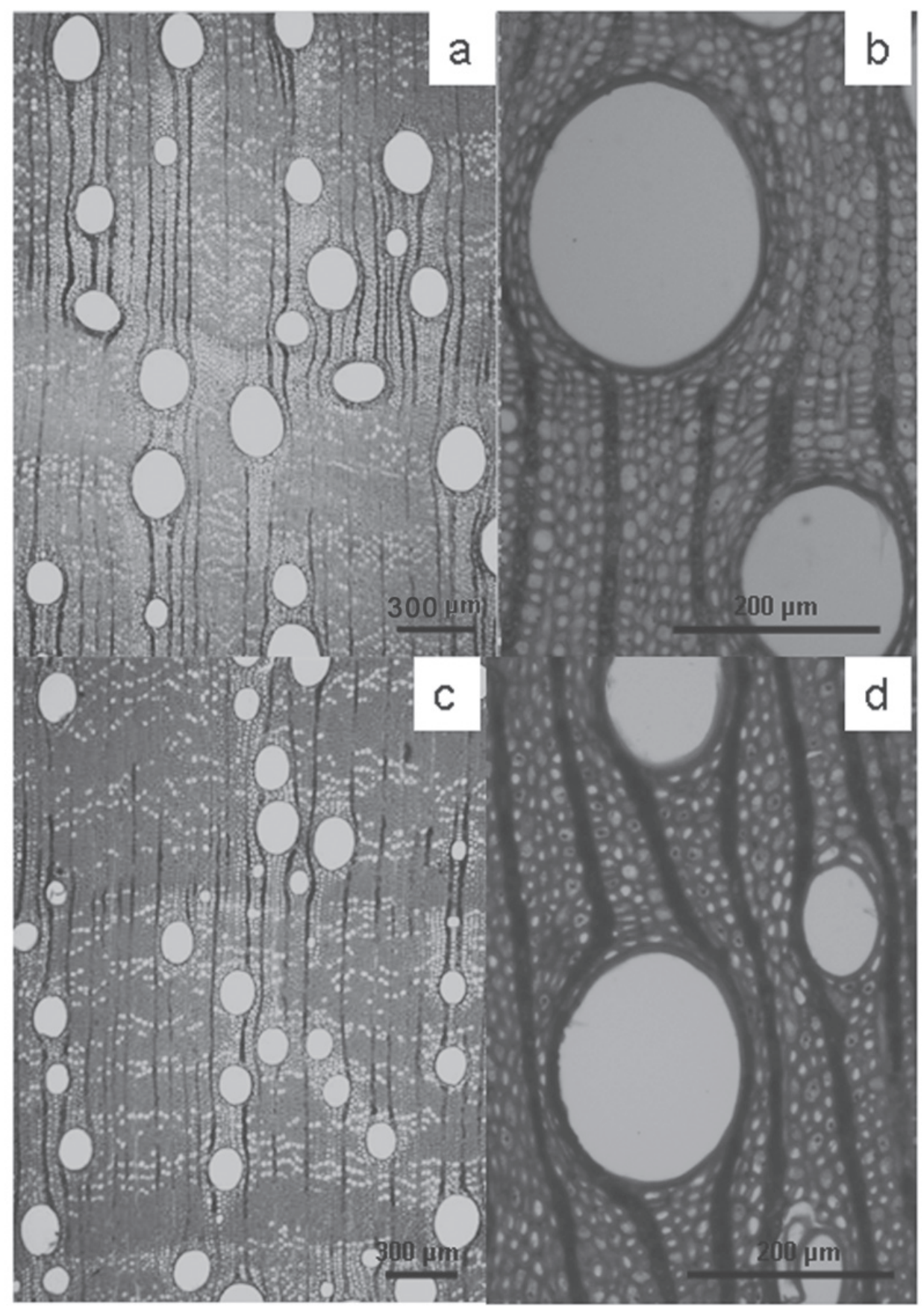

Figura 1. Vistas transversales de Quercus obtusata, características de anillos de crecimiento, porosidad y parénquima axial. a,b. Llano el Guilo, 338a Aguilar; c,d. Arcos del Sitio, 343a Aguilar. 


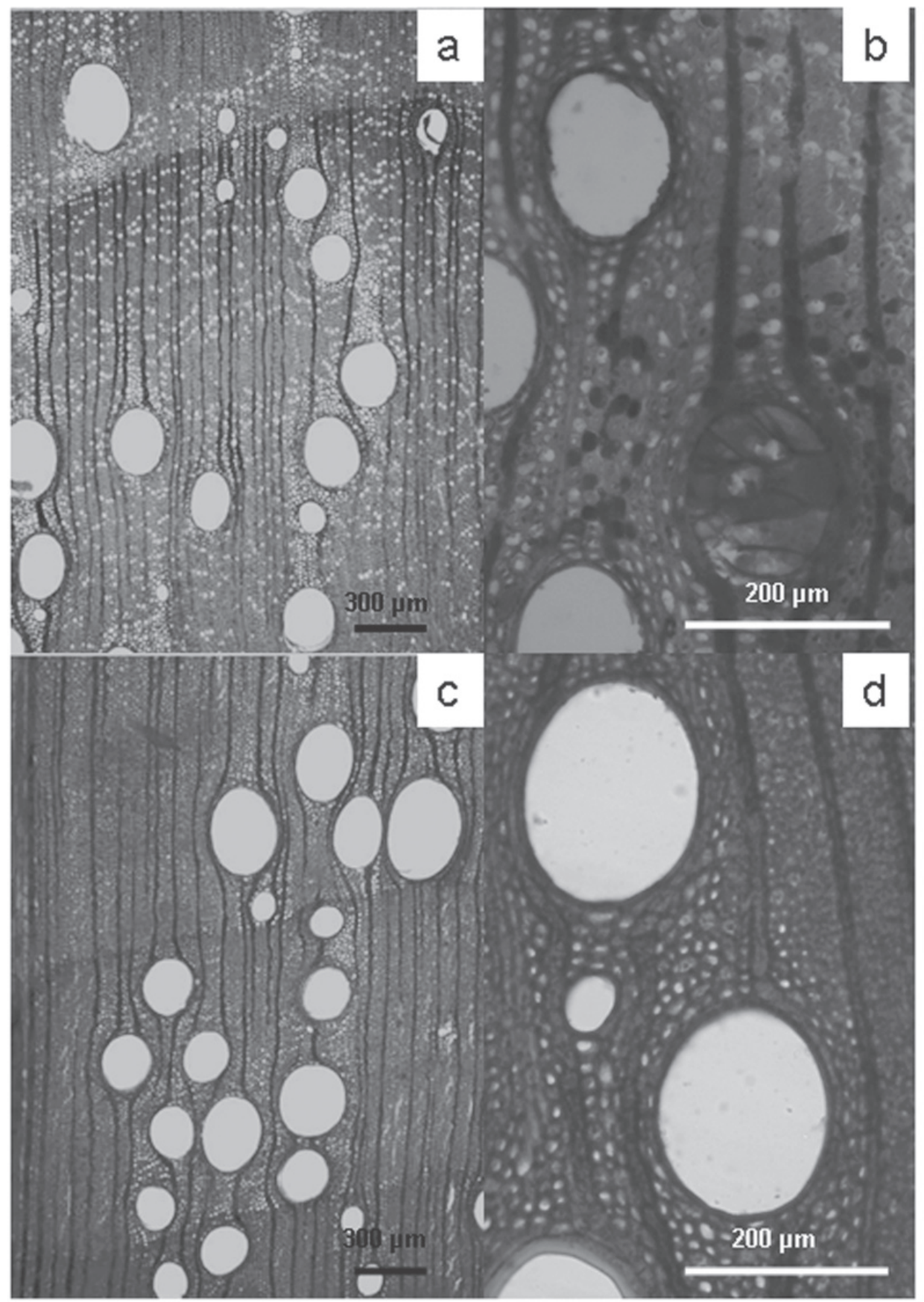

Figura 2. Características de anillos de crecimiento, porosidad y parénquima axial de Quercus obtusata, cortes transversales. a,b. Santa Catarina, 349 Aguilar; c,d. Santa Ana Jilotzingo, 350 Aguilar. 

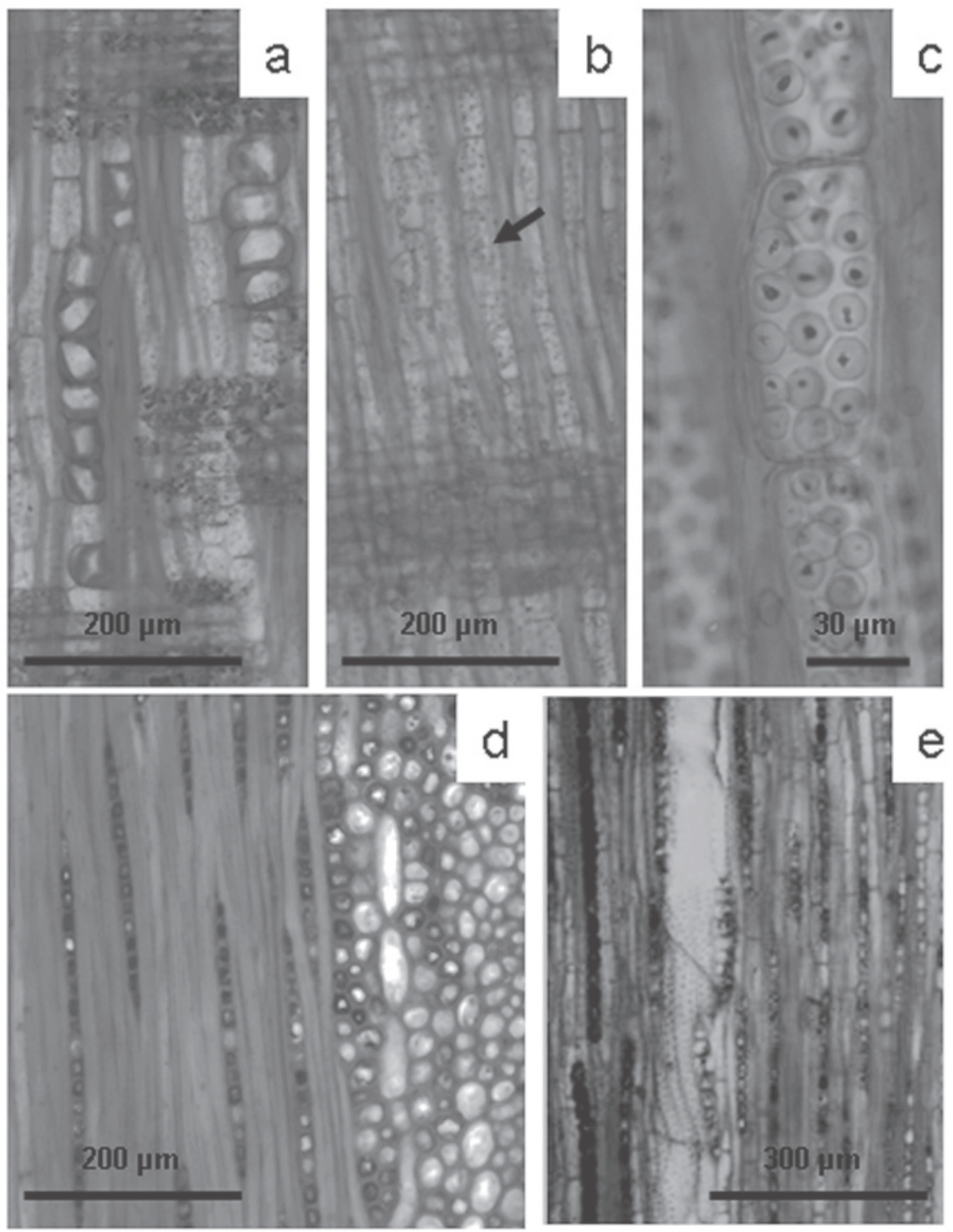

Figura 3. Características de radios de Quercus obtusata. a-c. Vistas radiales, Llano el Guilo. a. cristales prismáticos, 338; b, c. parénquima axial con almidón, 338b Aguilar; d, e. Vistas tangenciales. d. Llano el Guilo, 338b; e. Arcos del Sitio, 343a Aguilar. 


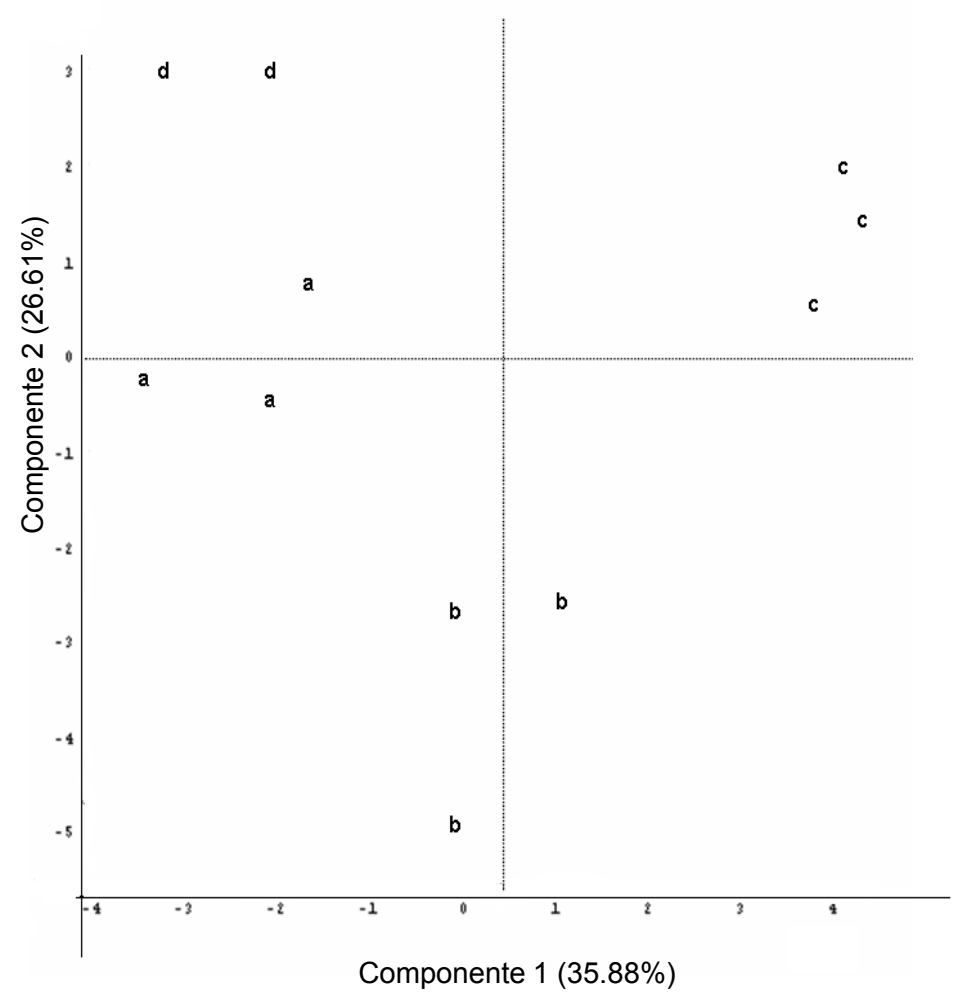

Figura 4. Representación gráfica del análisis de componentes principales de las poblaciones de Quercus obtusata colectadas en cuatro localidades. Los componentes 1 y 2 explican $62,49 \%$ de la variación total.

Análisis de varianza. El análisis de varianza no detectó diferencias significativas para los caracteres anatómicos entre las localidades $(>0,19 p<0,96)$, excepto para la altura de radios $(F=$ $3,88, g l=3, p<0,05)$, los cuales son más cortos en Llano el Guilo (Tabla 2) y la Relación Runkel $(F=15,28, g l=3, p<$ $0,001)$, siendo mayor en Arcos del Sitio (Tabla 2). Sin embargo, algunas tendencias pudieron apreciarse. Por ejemplo, la longitud de los elementos de vaso ancho y angosto más largos los presentó la localidad con mayor humedad, Santa
Catarina, con $621,31 \pm 109 \mu \mathrm{m}$ y 645,88 $\pm 82 \mu \mathrm{m}$ respectivamente; los elementos de vaso ancho más cortos, con 552,30 4 $73 \mu \mathrm{m}$ de longitud, los presentó la localidad de Santa Ana Jilotzongo, que se ubica a la mayor altitud de las poblaciones muestreadas. Los radios uniseriados más altos se presentaron en la localidad de Santa Ana Jilotzingo, con $515,43 \pm 121 \mu \mathrm{m}$, la población con mayor humedad, mientras que los radios más cortos con $326,71 \pm 126 \mu \mathrm{m}$, se presentaron en Llano el Guilo. 
Tabla 1. Características de los individuos, ubicación geográfica y variables climáticas de los sitios de colecta de Quercus obtusata. Oscilación diurna de la temperatura (ODT), isotermalidad (ISO), estacionalidad de la temperatura ( $E T)$, temperatura máxima promedio del periodo más cálido (TMMPC), temperatura mínima promedio del periodo más frío (TMPPF), temperatura promedio del periodo más lluvioso, (TPCLL), temperatura promedio del periodo cálido $(T P C)$, temperatura promedio del periodo más frío $(T P F)$, precipitación anual $(P A)$, precipitación promedio del periodo más cálido $(P C C)$, precipitación promedio del periodo más frío $(P C F)$.

\begin{tabular}{|c|c|c|c|c|}
\hline Variables/Localidades & $\begin{array}{l}\text { Llano el Guilo, } \\
\text { Estado de México }\end{array}$ & $\begin{array}{l}\text { Arcos del Sitio, } \\
\text { Estado de México }\end{array}$ & $\begin{array}{l}\text { Santa Catarina, } \\
\text { Morelos }\end{array}$ & $\begin{array}{l}\text { Santa Ana Jilotzingo, } \\
\text { Estado de México }\end{array}$ \\
\hline Identificación & a & $\mathrm{b}$ & c & $d$ \\
\hline Altura (m) & 131315 & 1518,611 & 241932 & 1519 \\
\hline$D A P(\mathrm{~cm})$ & $4336,533,5$ & 444734 & 644232 & 3129 \\
\hline Coordenadas & $19^{\circ} 42^{\prime} 44^{\prime \prime}$ & $19^{\circ} 41^{\prime} 05^{\prime \prime}$ & $19^{\circ} 00^{\prime} 51^{\prime \prime}$ & $19^{\circ} 33^{\prime} 1^{\prime \prime}$ \\
\hline $\mathrm{N} / \mathrm{W}$ & $99^{\circ} 27^{\prime} 37^{\prime \prime}$ & $99^{\circ} 12^{\prime} 21^{\prime \prime}$ & $99^{\circ} 09^{\prime} 43^{\prime \prime}$ & $99^{\circ} 24^{\prime} 1^{\prime \prime}$ \\
\hline Altitud (m s.n.m.) & 2525 & 2402 & 2220 & 2700 \\
\hline Tipo de vegetación & $\begin{array}{l}\text { Bosque de } \\
\text { encino-pino }\end{array}$ & $\begin{array}{l}\text { Matorral } \\
\text { xerófilo }\end{array}$ & $\begin{array}{l}\text { Bosque de encino } \\
\text { con elementos } \\
\text { mesófilos }\end{array}$ & $\begin{array}{l}\text { Bosque } \\
\text { de encino }\end{array}$ \\
\hline$O D T$ & 16,2 & 16,7 & 15,2 & 14,7 \\
\hline ISO & 0,68 & 0,67 & 0,68 & 0,68 \\
\hline$E T$ & 0,66 & 0,7 & 0,57 & 0,6 \\
\hline TMMPC & 25,8 & 27,1 & 28,2 & 24,2 \\
\hline TMPPF & 2 & 2,2 & 5,7 & 2,6 \\
\hline TPCLL & 15,8 & 17 & 18 & 14,8 \\
\hline$T P C$ & 16,6 & 17,7 & 19,2 & 15,8 \\
\hline TPF & 11,8 & 12,6 & 14,9 & 11,3 \\
\hline$P A$ & 886 & 721 & 996 & 1085 \\
\hline$P C C$ & 295 & 246 & 289 & 312 \\
\hline PCF & 31 & 22 & 30 & 38 \\
\hline
\end{tabular}




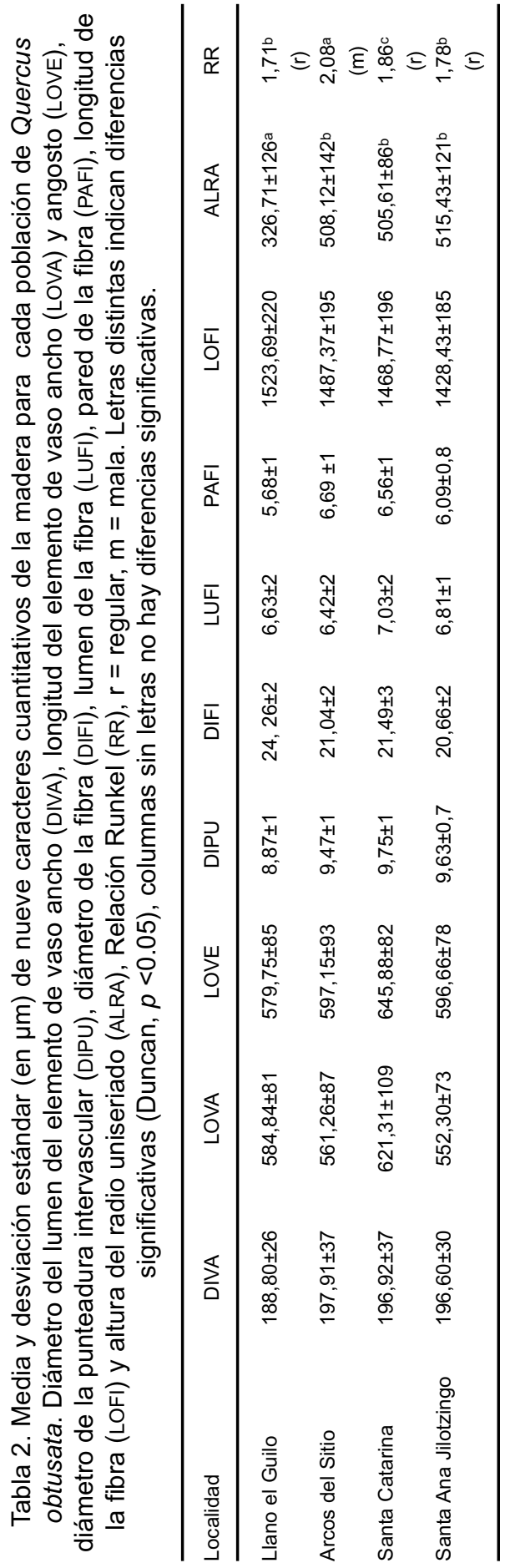

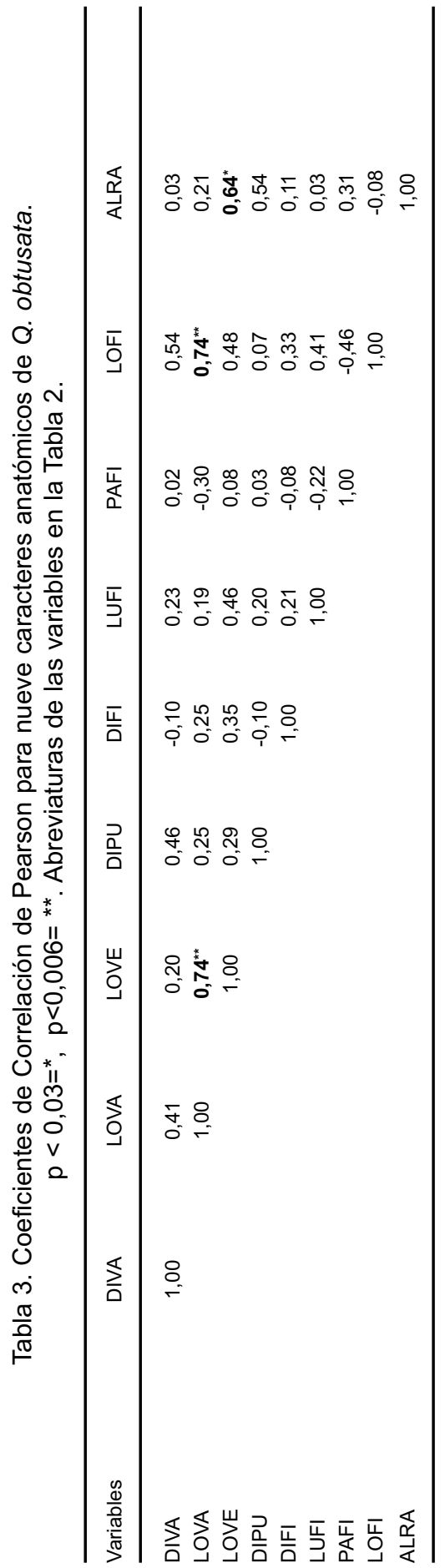


Tabla 4. Eigenvectores del análisis de componentes principales de los caracteres anatómicos de la madera de Quercus obtusata. Las variables con mayor carga marcadas en negritas. Altura del individuo (ALTURA in), abreviaturas de las variables en las Tablas 1 y 2 .

\begin{tabular}{cccc}
\hline Variables & PRIN 1 & PRIN 2 & PRIN3 \\
\hline DIVA & 0,031 & 0,035 & 0,252 \\
LOVA & 0,157 & 0,101 & $\mathbf{0 , 3 7 4}$ \\
LOVE & 0,239 & 0,122 & 0,161 \\
DIPU & 0,102 & 0,195 & 0,164 \\
DIFI & 0,190 & $-0,078$ & $-0,225$ \\
LUFI & 0,093 & 0,223 & 0,187 \\
PAFI & 0,143 & $-0,159$ & $-\mathbf{0 , 3 2 6}$ \\
LOFI & 0,038 & 0,017 & $\mathbf{0 , 5 2 4}$ \\
ALRA & 0,164 & 0,048 & $-0,099$ \\
RR & 0,050 & 0,243 & $-\mathbf{0 , 3 6 0}$ \\
ALTURA in & 0,254 & 0,168 & $-0,104$ \\
DAP & 0,270 & $-0,027$ & 0,023 \\
ALTI & $\mathbf{- 0 , 3 1 5}$ & 0,096 & $-0,044$ \\
ODT & $-0,057$ & $-0,356$ & 0,189 \\
ISO & $-0,019$ & 0,341 & $-0,053$ \\
ET & $-0,250$ & $-0,201$ & 0,167 \\
TMMPC & 0,305 & $-0,138$ & 0,058 \\
TMPPF & 0,292 & 0,174 & $-0,102$ \\
TPCLL & 0,313 & $-0,123$ & 0,045 \\
TPC & $\mathbf{0 , 3 2 6}$ & $-0,070$ & 0,019 \\
TPF & $\mathbf{0 , 3 2 9}$ & 0,008 & $-0,010$ \\
PA & 0,006 & $\mathbf{0 , 3 7 8}$ & $-0,146$ \\
PCC & $-0,084$ & $\mathbf{0 , 3 5 7}$ & $-0,084$ \\
PCF & $-0,103$ & $\mathbf{0 , 3 6 2}$ & -116 \\
\hline
\end{tabular}

\section{DISCUSIÓN}

Se encontró variación en las características de los anillos de crecimiento entre los individuos de Quercus obtusata. En Llano el Guilo y Santa Ana Jilotzingo, las bandas de fibras con paredes engrosadas que definen la madera tardía, son apenas perceptibles, mientras que en Santa Catarina, el grosor de la pared de las fibras en la banda disminuye de manera progresiva hacia la madera temprana. La formación y características de los anillos de crecimiento, pueden estar asociados con fenómenos ambientales, fisiológicos (Carquist, 1988), o relacionarse con el origen geográfico de las especies (Aguilar-Rodríguez y
Barajas-Morales, 2005); para el caso de Q. obtusata aspectos endógenos como los fisiológicos, podrían estar regulando las diferencias observadas. El parénquima axial también exhibe diferencias; a pesar de que aquí se describe como apotraqueal difuso y en agregados y paratraqueal vascicéntrico en la madera de individuos que crecen en Santa Ana Jilotzingo existe menor cantidad de parénquima axial que en el resto de las otras localidades. Esta variación también se observa al comparar lo reportado por otros autores para la misma especie (Aguilar-Rodríguez et al., 2000; De la Paz Pérez-Olvera, 1982, 1985; De la Paz Pérez-Olvera et al., 2006; Honorato, 2002; Ortega et al., 1988). 
Asociación entre caracteres anatómicos. La correlación entre caracteres anatómicos mostró que en la madera de $Q$. obtusata los coeficientes de correlación más altos y positivos existen entre la longitud de los elementos de vaso, la longitud de la fibra y la altura de los radios; fenómeno que puede explicarse por el origen común que estos elementos tienen en el cámbium vascular (Bailey, 1920; Metcalfe y Chalk, 1983), por lo que frecuentemente resultados similares se han obtenido para otros taxa (AguilarRodríguez y Terrazas, 2001; Carlquist y Hoekman, 1985; Moya y Tomazello, 2008). Sin embargo, en $Q$. obtusta ninguno de los caracteres de las fibras, como longitud, diámetro, lumen y pared, mostraron alguna asociación significativa entre ellos, a diferencia de lo registrado para otras especies (Aguilar-Rodríguez y Terrazas, 2001). Estos caracteres tampoco varían significativamente entre las diferentes localidades; aunque las fibras con paredes de mayor grosor se observan en la localidad más seca (Arcos del Sitio); estas diferencias no son estadísticamente significativas con las de Santa Catarina, que se caracteriza por tener una mayor humedad ambiental.

La asociación entre caracteres de las fibras ha sido utilizada para establecer ciertas propiedades de la madera; por ejemplo, en la elaboración de pulpa para papel caracteres como pared, diámetro, lumen y longitud de la fibra, permiten obtener algunos criterios sobre su calidad (Tamarit, 1996; Tamolang y Wangaard, 1961; Yáñez-Espinosa et al., 2004). Con base en esto la madera de algunas especies de Quercus ha sido propuesta para ser empleada en la elaboración de papel de buena y regular calidad (Honorato, 2002; Tamarit, 1996), por lo que registrar la variación en estas características puede aportar información para la elección de poblaciones con este fin. Lo anterior se ve reflejado de manera general en
Q. obtusata, pues haciendo un ensayo con respecto a los valores obtenidos de la Relación Runkel se observa que las fibras de calidad regular para la elaboración potencial de pulpa se encuentra en aquellas maderas que se desarrollan en poblaciones con mayor cantidad de precipitación al año como Llano el Guilo, Santa Ana Jilotzingo y Santa Catarina, mientras que fibras de mala calidad están presentes en maderas de árboles que crecen en ambientes como el de Arcos del Sitio, en donde se registra menor precipitación de lluvia, así como los individuos de menor talla.

Variación anatómica de la madera asociada con el clima. Numerosos trabajos han evaluado la relación que los caracteres anatómicos de la madera tienen con el clima (Aguilar-Rodríguez et al., 2006; Cordell et al., 1998; Dünisch y Bauch, 1994; Moya y Tomazello, 2008; Psaras y Sofroniou, 1999; Roig, 1986; Villagra y Roig, 1997; Villar-Salvador et al., 1997; Wiemann et al., 2001; YáñezEspinosa y Terrazas, 2001) y aunque se han propuesto tendencias ecológicas generales, el estudio de la variación de la madera a nivel intraespecífico es interesante, debido a que los resultados no siguen siempre un patrón típico y las diferencias entre las dimensiones de los elementos de vaso y fibras en las maderas provenientes de ambientes contrastantes llegan a ser evidentes (Aguilar-Rodríguez et al., 2006; Arias y Terrazas, 2001; Cordell et al., 1998; Roig, 1986; Noshiro y Baas, 2000; Villagra y Roig, 1997). Para el caso de Q. obtusata, el análisis de componentes principales reveló que la distribución de la temperatura y la precipitación a lo largo del año, así como el promedio total de lluvia al año, separaron las cuatro localidades de Q. obtusata. A pesar de que las poblaciones de esta especie se desarrollan principalmente en ambientes templados, el microclima en su área de distribución 
varía de un sitio a otro. En Santa Catarina, Morelos, se registró una precipitación anual de $996 \mathrm{~mm}$ y la temperatura del periodo más frío de $15^{\circ} \mathrm{C}$, que es la más alta de las cuatro poblaciones. En este sitio el lumen de las fibras y la longitud de los elementos de vaso mostraron sus mayores dimensiones con respecto a las demás poblaciones. Es sabido que la presencia de los elementos de vaso y fibras largos, con lúmenes amplios se relacionan con una elevada humedad (Aguilar-Rodríguez y BarajasMorales, 2005), aspecto que también se ha visto en las especies perennifolias Quercus coccifera y $Q$. ilex (VillarSalvador et al., 1997), donde la longitud de los elementos de vaso aumenta conforme hay una mayor precipitación, así como en Gmelina arborea para diámetro y lumen de la fibra (Moya y Tomazello, 2008). En la localidad más seca, Arcos del Sitio, con una precipitación promedio de $721 \mathrm{~mm}$ y temperatura anual de $13^{\circ} \mathrm{C}$, el grosor de la pared de las fibras tiende a ser mayor y su lumen a estar más reducido, así mismo, la longitud de los elementos de vaso ancho es más corta; éstos últimos también están reducidos en Santa Ana Jilotzingo, localizada a una mayor altitud (2700 m s.n.m.). En otros casos se ha reconocido la influencia negativa que la altitud tiene sobre algunos caracteres anatómicos, encontrándose que los cambios en la anatomía de la madera son parecidos a los que se producen por sequía o congelación (Fisher et al., 2007; Metcalfe y Chalk, 1983). Sin embargo, no siempre existen tendencias en todos los elementos celulares con respecto al clima, como es el caso en $Q$. obtusta para la longitud de las fibras y la altura de los radios uniseriados. Las fibras más largas se reportan para Llano el Guilo, población con características climáticas intermedias entre las localidades; probablemente, la mayor dimensión de las fibras en esta localidad está relacionada con el creci- miento intrusivo de las mismas, el cual puede comprender un tiempo de diferenciación más amplio durante la época favorable. Otro aspecto que llama la atención es que en Santa Ana Jilotzingo los radios uniseriados son los más altos, situación que quizá se asocie con la menor cantidad de parénquima axial presente en su madera, a diferencia de lo que se aprecia en las otras localidades. Respuestas poco claras de algunos elementos celulares con factores climáticos, latitudinales o altitudinales han sido observadas en Quercus faginea, especie caducifolia (Villar-Salvador et al., 1997); en Gmelina arborea la longitud de las fibras y altura de radios tampoco fueron significativas con los parámetro ecológicos estudiados (Moya y Tomazello, 2008), así como en especies de otros grupos taxonómicos (Liu y Noshiro, 2003; Noshiro y Baas, 2000). Al respecto Liu y Noshiro (2003) exponen que la variación en la anatomía de la madera a nivel de especie no siempre es controlada por gradientes ecológicos.

Variación anatómica de la madera asociada con el hábito. Con respecto al hábito de los individuos de Quercus obtusata sólo se encontró una correlación significativa y positiva con la longitud del elemento de vaso angosto $(\leq 150 \mu \mathrm{m})$. Es común esperar que conforme aumenta la talla de los individuos los elementos celulares también incrementen su tamaño (Aguilar-Rodríguez et al., 2006; Arias y Terrazas, 2001; Noshiro y Baas, 1998, 2000; Terrazas et al., 2008), como se observa en Cornus controversa, donde existe una relación positiva entre la longitud del elemento de vaso y la altura y diámetro del tallo (Noshiro y Baas, 2000). Sin embargo, la longitud de las fibras en Q. obtusata no mostró alguna asociación significativa con la altura de los individuos, aspecto poco común, pues en general las dimensiones de las fibras, en especial su longitud, aumentan con rela- 
ción al tamaño del individuo como se ha observado en Pachycereus pecten-aboriginum, Buddleja cordata y Gmelina arborea (Aguilar-Rodríguez et al., 2006; Arias y Terrazas, 2001; Moya y Tomazello, 2008). No obstante, comparando los datos obtenidos en este estudio con lo publicado por Aguilar-Rodríguez et al. (2000) y De la Paz Pérez-Olvera (1985), la longitud de las fibras de $Q$. obtusata es mayor en individuos más altos $(20 \mathrm{~m})$, en los cuales se reporta una longitud promedio para las fibras de $1880 \mu \mathrm{m}$ y $1696 \mu \mathrm{m}$ respectivamente. Aunque existen varios trabajos donde se aporta información sobre estos elementos, no siempre se pueden comparar con la talla de los individuos por la falta de información en los trabajos consultados.

En Q. obtusata existe una correlación positiva y significativa entre el tamaño y diámetro de los individuos y negativa con el gradiente altitudinal, observándose que árboles de menor talla y diámetro se localizaron en Santa Ana Jilotzingo, zona de mayor altitud, en contraste con los que crecen en Santa Catarina, en donde la altitud es menor. Esto concuerda con las tendencias generales propuestas para otras especies, como Metrosideros polymorpha y Rhododendron caucasicum (Cordell et al., 1998; Merev y Yavuz, 2000).

\section{CONCLUSIONES}

En la madera de $Q$. obtusata se detectó una respuesta significativa con algunas de las variables climáticas, que pueden afectar la calidad de la pulpa de madera. Por ello, se deberán tomar en cuenta estos resultados en programas de restauración ecológica o reforestación con fines de aprovechamiento. Los estudios sobre variación anatómica en la madera a nivel de especie muestran resultados contrastantes, consecuencia de condiciones ambientales de cada localidad. Por lo que para sugerir un uso adecuado para la especie se debe poner atención en su procedencia microclimática, pues ésta influye directamente en el comportamiento de los elementos celulares.

\section{RECONOCIMIENTOS}

Agradecemos a la Dra. Silvia Romero Rangel y $M$. en C. E. Carlos Rojas Zenteno, del laboratorio de árboles y arbustos de la FES Iztacala UNAM, la selección e identificación de los individuos en el campo. Este estudio fue financiado a través del Programa de Apoyo a los Profesores de Carrera para la formación de Grupos de Investigación PAPCA 20032004, de la FES Iztacala, UNAM y el análisis y escritura de este manuscrito se concluyo durante el periodo sabático de SAR.

\section{REFERENCIAS}

Aguilar-Rodríguez, S. y B. J. CastroPlata. 2006. Anatomía de la madera de doce especies del bosque mesófilo de montaña del Estado de México. Madera y Bosques 12(1): 95-115.

Aguilar-Rodríguez, S., J. Barajas-Morales y J. D. Tejero-Díez. 2000. Anatomía de maderas de México: especies de un bosque mesófilo de montaña. Instituto de Biología, UNAM 87 p.

Aguilar-Rodríguez, S., y J. BarajasMorales. 2005. Anatomía de la madera de especies arbóreas de un bosque mesófilo de montaña: un enfoque ecológico-evolutivo. Boletín de la Sociedad Botánica de México 77:51-58.

Aguilar-Rodríguez, S. y T. Terrazas. 2001. Anatomía de la madera de Buddleja 
L. (Buddlejaceae): análisis fenético. Madera y Bosques 7(2):63-85.

Aguilar-Rodríguez, S., T. Terrazas y L. López Mata. 2006. Anatomical wood variation of Buddleja cordata (Buddlejaceae) along its natural range in Mexico. Trees 20:253-261.

Arias, S. y T. Terrazas, 2001. Variación en la anatomía de la madera de Pachycereus pecten-aboriginum (Cactaceae). Anales del Instituto de Biología. Universidad Nacional Autónoma de México, Serie Botánica 72(2):157-169.

Bailey, I. W. 1920. The cambium and its derivative tissues. II Size variations of cambium initials in gymnosperms and angiosperms. American Journal of Botany 7:355-367.

Berlyn, G. P. y J. P. Miksche. 1976. Botanical microtechnique and cytochemistry. lowa State University Press. lowa. $326 \mathrm{p}$.

Busby, J. R. 1986. Bioclimatic Prediction System (BIOCLIM) User's Manual version 2.0 Australian Biological Resources Study Leaflet.

Carlquist, S. 1988. Comparative wood anatomy. Systematic, ecological, and evolutionary aspects of dicotyledon wood. Springer-Verlag. Berlin. $436 \mathrm{p}$.

Carlquist, S. y D. A. Hoekman. 1985. Ecological wood anatomy of the woody southern Californian flora. International Association of Wood Anatomists Bulletin new series 6:319-347.

Cordell, S., G. Goldstein., D. Mueller, D. Webb y P. M. Vitousek. 1998. Physiological and morphological variation in Metrosideros polymorpha, a dominant Hawaiin tree species, along an altitudinal gradient: the role of phenotypic plasticity. Oecologia 113:188- 196.

Corral, L. 1981. Anatomía de la madera de siete especies del género Quercus. Boletín divulgativo del Instituto Nacional de Investigaciones Forestales, núm. 73. México, D.F. $45 \mathrm{p}$.

De la Paz Pérez-Olvera, C. 1976. Características anatómicas de cinco encinos de México. Boletín Técnico del Instituto Nacional de. Investigaciones Forestales, núm. 46. México, D.F. 33 p.

De la Paz Pérez-Olvera, C. 1982. Anatomía de la madera de cinco especies de encinos de Durango. Boletín Técnico del Instituto Nacional de. Investigaciones Forestales núm. 43. México, D.F. 35 p.

De la Paz Pérez-Olvera, C. 1985. Características anatómicas de siete especies del género Quercus. Boletín Técnico del Instituto Nacional de. Investigaciones Forestales, núm. 123. México, D.F. 70 p.

De la Paz Pérez-Olvera, C. y R. DávalosSotelo. 2008. Algunas características anatómicas y tecnológicas de la madera de 24 especies de Quercus (encinos) de México. Madera y Bosques 14(3):43-80.

De la Paz Pérez-Olvera, C. y A. Quintanar I. 1994. Características anatómicas de la madera de cinco especies del estado de Jalisco, México. Acta Botánica Mexicana 27:75-87.

De la Paz Pérez-Olvera, C., A. Campos R., A. Quintanar I. y R. DávalosSotelo. 1998. Estudio anatómico de la madera de cinco especies del género Quercus (Fagaceae) del estado de Veracruz. México. Madera y Bosques 4(2):45-65. 
De la Paz Pérez-Olvera, C., R. DávalosSotelo. y E. Guerrero C. 2000. Aprovechamiento de la madera de encino en México. Madera y Bosques 6(1):3-13.

De la Paz Pérez-Olvera, C., S. Vélez J. y J. Ceja R. 2006. Anatomía de la madera de ocho especies de Quercus (Fagaceae) de Oaxaca, México. Madera y Bosques 12(1): 63-94.

Dünisch, O y J. Bauch. 1994. Influence of mineral elements on wood formation of old growth spruce (Picea abies [L.] Karst). Holzforcechung 48:5-14.

Fisher, B. J., G. Goldstein, T. J. Jones y S. Cordell. 2007. Wood vessel diameter is related to elevation and genotype in the Hawaiian tree Metrosideros polymorpha (Myrtaceae). American Journal of Botany 94:709-715.

García, E. 2004. Modificaciones al Sistema de Clasificación Climática de Köppen. 5a . Ed. Instituto de Geografía. Universidad Nacional Autónoma de México. Serie libros núm. $6.90 \mathrm{p}$.

Honorato, S. J. A. 2002. Índices de calidad de pulpa y papel de encinos mexicanos. In: J. Quintanar O, ed. Características, propiedades y procesos de transformación de la madera de los encinos de México. INIFAP. Centro de Investigación Regional del Centro Campo experimental San Martinito. Puebla. p: 107-121.

Honorato, S. J. A. y M. E. Fuentes L. 2001. Propiedades físico-mecánicas de la madera de cinco especies de encino del estado de Guanajuato. Ciencia Forestal 90:5-28.

IAWA Committee. 1989. International Association of Wood Anatomists list of microscopic features for hardwood identification. International Associa- tion of Wood Anatomists Bulletin new series 10:219-332.

Johansen, J. 1940. Plant microtechnique. Mc Graw-Hill Book Co. Nueva York. $523 \mathrm{p}$.

Liu, J. y S. Noshiro. 2003. Lack of latitudinal trends in wood anatomy of Dodonaea viscosa (Sapindaceae), a species with a worldwide distribution. American Journal of Botany 90:532539.

Martínez, C. D., T. Terrazas y F. Zavala Ch. 2003. Arquitectura foliar y anatomía de la corteza y la madera de Quercus Sartorii y Quercus Xalapensis (Fagaceae). Boletín de la Sociedad Botánica de México 73:62-72.

Metcalfe, C. R. y L. Chalk. 1983. Anatomy of the dicotyledons, vol. II. 2 ed. Clarendon Press, Oxford. 297 p.

Merev, N. y H. Yavuz. 2000. Ecological wood anatomy of Turkish Rhododendron L. (Ericaceae). Intraspecific variation. Turkish Journal of Botany 24:227-237.

Moya, R. y M. Tomazello F. 2008. Variation in the wood anatomical structure of Gmelina arborea (Verbenaceae) trees at different ecological conditions in Costa Rica. Revista de Biología Tropical 56(2):689-704.

Nikon Corporation. 1991-2006. NISElements, Versión 2.33.

Noshiro, S. y P. Baas. 1998. Systematic Word anatomy of Cornaceae and allies. International Association of Wood Anatomists Journal 19:43-97.

Noshiro, S. y P. Baas. 2000. Latitudinal trends in wood anatomy within species and genera: Case study in Cornus s.l. (Cornaceae). American Journal of Botany 87:1495-1506. 
Ortega, F., L. Guerrero, T. Carmona y C. Córdoba. 1988. Anatomía de la madera de veintiocho especies de Cosautlán de Carvajal, Veracruz. La madera y su uso Boletín Técnico, núm. 19. INIREB- LACITEMA. México. 206 p.

Psaras, G.K. y I. Sofroniou.1999. Wood anatomy of Capparis spinosa from an ecological perspective. International Association of Wood Anatomists Journal 20:419-429.

Quintanar, O. J. 2002. Aprovechamiento forestal y usos de los encinos de México. In:J. Quintanar O, ed. Características, propiedades y procesos de transformación de la madera de los encinos de México. INIFAP. Centro de Investigación Regional del Centro Campo experimental San Martinito. Puebla. p: 21-33.

Roig, J. F. A. 1986. The wood of Adesmia horrida and its modifications by climatic conditions. International Association of Wood Anatomists Bulletin 7:129-135.

Romero, R. S., E. C. Rojas Z. y E. Aguilar. 2002. El género Quercus (Fagaceae) en el Estado de México. Annals of the Missouri Botanical Garden 89(4):551-593.

Rzedowski, J. 1978. Vegetación de México. Limusa. México, D.F. 472 p.

SAS Institute. 1989. SAS user's guide statistics. Cary, North Carolina.

Tamarit, U. J.C. 1996. Determinación de los índices de pulpa para papel de 132 maderas latifoliadas. Madera y Bosques 2(2):29-41.

Tamolang, F. N. y F. F. Wangaard. 1961. Relationships between harwood fiber characteristics and pulpsheet properties. Tappi 44(3):201-216.

Terrazas, T y S. Loza-Cornejo. 2003. Anatomía de la madera y alometría de veinte especies de Stenocereus (Cactaceae). Anales del Instituto de Biología Universidad Nacional Autónoma de México, Serie Botánica 74(2):195-208.

Terrazas, T., S. Aguilar-Rodríguez y L. López-Mata. 2008. Wood anatomy and its relation to plant size and latitude in Buddleja L. (Buddlejaceae). Interciencia 33(1):46-50.

Valencia, A. S. 2004. Diversidad del género Quercus (Fagaceae) en México. Boletín de la Sociedad Botánica de México 75:33-53.

Valencia, A. S. y J. Barajas-Morales, 1995. Comparación anatómica de la madera de Quercus affinis y $Q$. laurina (Fagaceae). Anales del Instituto de Biología, Universidad Nacional Autónoma de México, Serie Botánica 66(2):113-131.

Villar-Salvador, P., P. Castro-Díez, C. Pérez -Rontomé y G. MontserratMartí. 1997. Stem xylem features in three Quercus (Fagaceae) species along a climatic gradient in NE Spain. Trees 12: 90-96.

Villagra, P. y F. A. Roig J. 1997. Wood structure of Prosopis alpataco and $P$. argentina growing under different edaphic conditions. Internacional Association of Wood Anatomists Journal 18:37- 51.

Wiemann, C. M., D. L. Dilcher y S. R. Manchester. 2001. Estimation of mean annual temperature from leaf and wood physionomy. Forest Science 47(2):141-149. 
Yáñez-Espinosa, L. y T. Terrazas. 2001. Variación anatómica en la madera y en la corteza de Annona glabra L. bajo inundación. Agrociencia 34:51-63.
Yáñez-Espinosa, L., T. Terrazas, L. López-Mata y J. I. ValdezHernández. 2004. Wood variation in Laguncularia racemosa and its effect in fibre quality. Wood Science and Technology 38:217-226. anatómica en la madera de Quercus obtusata (Fagaceae). Madera y Bosques 16(2):69-87. 